\title{
Collaboration et construction identitaire d'une étudiante chercheuse au doctorat en éducation
}

Josée Lachance et Jean-François Desbiens

\section{OpenEdition}

Édition électronique

URL : https://journals.openedition.org/ripes/3554

DOI : 10.4000/ripes.3554

ISSN : 2076-8427

Éditeur

Association internationale de pédagogie universitaire

Référence électronique

Josée Lachance et Jean-François Desbiens, « Collaboration et construction identitaire d'une étudiante chercheuse au doctorat en éducation », Revue internationale de pédagogie de l'enseignement supérieur [En ligne], 37(3) | 2021, mis en ligne le 09 novembre 2021, consulté le 27 septembre 2022. URL : http:// journals.openedition.org/ripes/3554; DOI : https://doi.org/10.4000/ripes.3554

Ce document a été généré automatiquement le 27 septembre 2022.

Creative Commons - Attribution - Pas d'Utilisation Commerciale - Partage dans les Mêmes Conditions 4.0 International - CC BY-NC-SA 4.0

https://creativecommons.org/licenses/by-nc-sa/4.0/ 


\title{
Collaboration et construction identitaire d'une étudiante chercheuse au doctorat en éducation
}

\author{
Josée Lachance et Jean-François Desbiens
}

Remerciement : J'aimerais remercier l'ensemble des membres de l'équipe : Pierre Paillé, JeanFrançois Desbiens et Marianne Xhignesse. J'en profite aussi pour exprimer toute ma gratitude à Pierre Vermersch, décédé en juillet 2020, pour son enseignement, son accompagnement et son amitié. Sa bienveillance, sa confiance et sa générosité ont contribué grandement à mes apprentissages et à mon enseignement de l'entretien d'explicitation. Nous aimerions également souligner l'apport de Maurice Legault qui a eu la gentillesse de bien vouloir animer l'entretien de groupe.

\section{Introduction}

1 La formation d'étudiants aux études supérieures est une entreprise de longue haleine. Ce cheminement rencontre deux principaux problèmes (Jutras et al., 2010) : le taux élevé d'abandon et la durée des études. À cet égard, Jones est d'avis (2013), que la relation entre l'étudiant et le directeur est d'une importance capitale tout en ajoutant que celle-ci «is likely to make or break the doctoral candidature» (p.92). La dynamique qui s'établit entre ces deux acteurs influe donc considérablement sur la réussite des études supérieures.

2 L'étude de Bravo et al. (2007) indique que la satisfaction des étudiants des études supérieures est en lien avec deux principaux facteurs: 1) l'implication du directeur dans la conception et la réalisation du projet de recherche et 2) la relation étudiantdirecteur. Ils considèrent l'encadrement de doctorants comme une relation dyadique. Ainsi, il semble que la relation entre l'étudiant et son directeur ou son équipe d'encadrement soit une condition de satisfaction et de réussite des études supérieures. 
3 Cet article décrit les principales étapes qui, au travers de la relation qui s'est établie avec son équipe d'encadrement, ont mené la première autrice de cet article à se construire comme candidate doctorale capable d'autonomie, de créativité et de pertinence scientifique. Il relatera des moments plus critiques où elle s'est interrogée sur la poursuite de son cheminement en thèse et il identifiera un point de bascule bien défini dans le temps qui lui a permis de se révéler comme chercheuse autonome.

Dans cet article, la problématique soulève l'importance de la dimension relationnelle dans le parcours au doctorat. Quant aux objectifs, ils ont trait à la description des différentes phases de collaboration, soit celles d'apprenti, de période intermédiaire de transformation, de compagnon et de maître (Narcy-Combes, 2001) ainsi qu'à l'émergence de l'autonomie de l'étudiante. Par la suite, le cadre conceptuel précise les concepts de collaboration et d'accompagnement, et ce, plus particulièrement dans le contexte des études supérieures. Ensuite, il est question des méthodes utilisées : l'autoexplicitation et l'entretien avec les membres de l'équipe d'encadrement, pour avoir accès à l'expérience vécue par l'étudiante ainsi qu'au contexte dans lequel la recherche a été effectuée. Les résultats exposent les étapes importantes de la collaboration et, plus spécifiquement, celles liées au moment critique qu'a été l'analyse qualitative. Les conditions nécessaires à l'autonomisation de l'étudiante sont aussi abordées. La discussion conclusive permet d'engager un dialogue entre les auteurs consultés quant à la dimension relationnelle au travers des points clés que sont les " positions de savoir ", la collaboration « dynamique vivante » et les conditions d'accompagnement.

\section{Problématique}

5 La qualité de l'encadrement a été mise sous le feu des projecteurs et la complexité des apprentissages dans l'encadrement des doctorants n'a été explorée qu'assez récemment (Turner, 2015). Plusieurs études tentent de mieux saisir l'apprentissage de l'encadrement (Amundsen et McAlpine, 2009; Turner, 2015) ou les pratiques d'encadrement des professeurs (Lee, 2008) en cherchant à décrire leur expérience. Un des appuis à leur apprentissage est sans contredit leur propre expérience du parcours au doctorat qui influence leurs pratiques. Amundsen et McAlpine (2009) proposent même de reconceptualiser l'apprentissage de l'encadrement sur un continuum qui s'étend du début des études doctorales jusqu'aux premières expériences d'encadrement à titre de professeur. Peu d'études décrivent spécifiquement le vécu singulier des doctorants durant leur étude. Pourtant, s'ils acceptaient de le partager en totalité ou en partie cela pourrait constituer un guide pour ceux qui s'engagent dans cette voie (Turner, 2015).

6 Lee (2008) propose cinq approches de supervision : fonctionnelle, d'enculturation, de pensée critique, d'émancipation et de développement d'une relation de qualité. Dans notre étude, nous nous centrons sur le développement de la qualité de la relation. Plus privée, la relation (Amundsen et McAlpine, 2009) est personnalisée (Turner, 2015) et influence directement le style d'encadrement (Amundsen et McAlpine, 2009). Turner (2015) identifie la dynamique relationnelle comme l'un des défis de l'encadrement au doctorat; cela exige de la persévérance et de la résilience pour l'encadreur. Lee (2008) invite à explorer plus avant cette relation d'encadrement, malgré les résistances compréhensibles et les questions éthiques qu'elle suppose. Cela permettrait d'éclairer des aspects demeurés dans l'ombre comme la transformation personnelle (Brew, 2001), 
l'inconfort vécu des doctorants confrontés aux opinions diverses des membres de son équipe d'encadrement (Guerin et Green, 2015) et l'établissement d'un climat de confiance réciproque conduisant au partage du pouvoir et de la responsabilité entre l'étudiant et son ou ses encadreurs (Robertson, 2017b).

7 À notre connaissance, peu de recherches ont été menées dans le but de démystifier les étapes importantes du parcours aux études doctorales et le type de relation qui s'instaure entre la doctorante et ses encadreurs. Cette recherche rapporte l'expérience vécue par une étudiante laquelle a été enrichie par les perspectives de tous les membres de son équipe d'encadrement.

\section{Objectifs de l'article}

Le présent article vise à identifier et décrire 1) les phases de collaboration importantes telles que vécues par une doctorante en éducation dans le contexte québécois; 2) les caractéristiques de l'encadrement collaboratif dont elle a bénéficié et 3) les conditions qui ont permis à l'étudiante de vivre un point de bascule.

\section{Cadre conceptuel}

9 Cette section débute par une présentation des notions de collaboration et d'accompagnement. Ces développements nous amènent par la suite à les approfondir à l'intérieur d'une réflexion sur la notion d'encadrement et ses différentes déclinaisons.

\subsection{Collaboration}

La collaboration est présente lorsque plusieurs personnes travaillent ensemble à la poursuite d'un objectif et d'un projet commun. Dans cet esprit, la recherche doctorale peut représenter l'aboutissement d'un processus où la contribution à divers degrés de tous les membres de l'équipe est requise et mise à profit dans la réalisation d'une œuvre commune (Robertson, 2017a). L'objectif de la collaboration est centré sur l'élaboration, l'avancement et la finalisation du projet de recherche de l'étudiante. Dans cette entreprise collaborative, tous les acteurs n'ont pas une posture symétrique avec un certain degré variable d'interdépendance. La véritable collaboration se reconnaît par l'engagement dans des projets de sens dans le cadre desquels chaque membre de l'équipe peut actualiser sa pratique et en retirer de la satisfaction (Little, 1990).

11 Ce type de collaboration ne correspond pas à l'idée de la recherche collaborative en éducation selon laquelle le praticien et le chercheur vont travailler ensemble à l'investigation et à la construction d'un projet de recherche (Desgagné, 1997). En effet, le milieu professionnel n'est pas investi dans la perspective que nous adoptons. En revanche, la combinaison "recherche» et «formation»y est bien présente. L'étudiante au doctorat est engagée dans un projet de recherche et tout le processus d'encadrement a pour but de développer sa capacité à mener de front les différentes opérations requises en vue de la réaliser. La manière d'établir et de vivre la relation dans l'équipe soulève inévitablement « la question du statut que chacun accorde à son propre savoir et au savoir de l'autre » soit la "position de savoir» (Morrissette et Desgagné, 2009, p. 139). Ce statut a eu une incidence sur « le pouvoir de contribution à 
la coconstruction du savoir, incluant l'établissement d'un rapport plus ou moins égalitariste ou hiérarchique, plus ou moins assujettissant ou affranchissant" (Morrissette et Desgagné, 2009, p. 140) mais ne doit jamais faire perdre de vue la visée transformative qui est poursuivie au travers de cet exercice.

L'autonomie est une finalité située au cœur du parcours doctoral en ce qu'il s'agit de «mener de façon autonome et experte un projet de recherche pour apporter une contribution originale à la connaissance " (Association des doyens des études supérieures au Québec [ADÉSAQ], 2018, p. 6). Selon Gohier et al. (2001), l'autonomie entretient un lien direct avec l'identité laquelle : « résulte de déséquilibres successifs et non d'une stabilité inaltérable. Elle ne consiste pas en la réitération du même, d'un modèle figé, mais dans la trajectoire d'un individu à travers les différents visages qu'elle peut prendre.» (p. 29). L'enjeu est d'établir collectivement au sein de l'équipe d'encadrement des conditions permettant à l'étudiant d'apprendre et de se construire comme un sujet autonome. La relation de type collaborative est particulière, car la doctorante est en voie d'enculturation de même qu'en quête d'émancipation (Lee, 2008), tout en étant en formation à la recherche par la recherche (Gérard, 2009). Ainsi, parfois, cette relation peut osciller selon ses besoins entre supervision et accompagnement. La supervision consiste en un rapport basé sur l'évaluation et la mise en marche des différentes étapes nécessaires pour répondre aux critères du programme de formation. L'accompagnement est plutôt centré sur des temps de réflexion avec l'étudiant afin de lui permettre d'élargir sa connaissance, tout en lui apportant du soutien.

\subsection{Accompagnement}

13 La démarche d'accompagnement "peut être définie comme "un » processus dynamisant trois logiques: relationnelle, spatiale et temporelle» (Paul, 2002, p. 54). L'accompagnement est protéiforme. Il a de multiples visages. Beauvais (2004) indique qu' « accompagner l'autre en tant que "sujet" revient alors à l'appréhender en tant que personne singulière, personne qui se construit, qui se "pro-jette", qui agit et s'assume dans un environnement donné » (p. 103).

14 Selon Paul (2002), le processus d'accompagnement n'est pas linéaire ni séquentiel. Il répond à une logique de mouvement avec des incertitudes. Ensuite, la qualité relationnelle est à trouver selon la dynamique en cours et est évolutive. Enfin, la pluralité des rôles s'adapte aux besoins. L'autonomie semble pour l'auteure un "produit même de ce dynamisme relationnel » (p. 54). Avec l'autonomie vient aussi la responsabilité. Beauvais (2004) parle ici de la « "méta-responsabilité", par ailleurs seule garante de sa première responsabilité envers l'autre, responsabilité ontologique, responsabilité de tout être humain impliqué dans une relation intersubjective " (p. 108). L'accompagnateur cherche à maintenir la juste distance relationnelle, à « réajuster » en permanence selon les enjeux du moment (Beauvais, 2004). Beauvais (2004) se réfère à cette dynamique vivante: "Accompagner l'autre, c'est faire un bout de chemin avec lui vers "quelque chose", ce bout de chemin se construit en marchant et ce "quelque chose" s'invente en se faisant » (p. 104). Cette citation permet de bien illustrer la part d'indétermination dans l'accompagnement et la nécessité de l'incertitude pour aider l'autre à se mettre en doute afin de trouver la voie de se réinventer tout en 
gardant de l'espace à l'imprévisible (Beauvais, 2004). L'accompagnateur cherche la juste dose d'incertitude pour créer l'ouverture sans amener l'autre vers la désorganisation.

Gérard (2009) apparente la relation pédagogique à celle de l'accompagnement sur le chemin des études. Trois postures avec autonomie variable pour l'étudiant sont évoquées: celle de conduite avec le directeur "devant»(sans autonomie), celle d'accompagnement-conseil avec le directeur "à côté » (semi-autonomie) et celle d'accompagnement-suivi avec le directeur «derrière»(pleine autonomie). Nous présentons quelques recherches en lien direct avec l'encadrement d'étudiants aux études supérieures.

\subsection{Réflexions sur l'encadrement de doctorants}

Styles et Radloff (2001) argumentent en faveur d'un modèle de supervision basé sur l'autorégulation de l'apprentissage. "Self-regulation is "the process by which an individual exercises control over the direction, persistence and intensity of thinking, affect and behaviour for the purpose of goal attainment" » (Kanfer et Kanfer, 1991, cité dans Styles et Radloff, 2001, p. 98). La régulation est faite par un processus métacognitif et engage l'apprenant dans son apprentissage. Autant l'étudiant que le superviseur sont engagés dans le processus d'autorégulation (Styles et Radloff, 2001). En plus de la régulation dans l'apprentissage, il est intéressant de regarder le processus par lequel l'étudiant se construit au fur et à mesure de sa formation doctorale.

Prenant appui sur l'exemple du diplôme d'études avancées (DEA) selon l'ancienne structure de formation européenne, Narcy-Combes (2001) identifie trois phases dans les différents statuts que comporte le processus d'encadrement d'un doctorant. À la première phase, l'étudiant est davantage un apprenti, qui a encore peu d'initiative sur le plan de la recherche. À la seconde phase, le processus de la thèse s'apparente à un voyage initiatique où le compagnon découvre diverses théories et « rencontre le monde pour s'ouvrir aux différences dans le respect des conventions du métier " (NarcyCombes, 2001, s.p.). Ce cheminement mène à la troisième phase où l'étudiant devient un maitre sans doctrine qui l'amène à se développer avec créativité, dans le respect des normes scientifiques en vigueur dans son domaine.

18 Le contexte de la recherche installe progressivement une approche horizontale des relations entre apprentis, compagnons et maîtres dans l'esprit d'une complémentarité des personnes. Cette perspective rejoint le mode de travail coopératif tout en privilégiant les talents et compétences de chacun (Narcy-Combes, 2001). Il y a un rapport hiérarchique entre le chercheur et le doctorant car ce dernier se retrouve en dessous. Selon que l'étudiant soit dans la phase d'apprenti, de compagnon ou de maître, la relation ainsi que la distribution de la responsabilité au sein de l'équipe connaîtront des degrés variables d'asymétrie.

Robertson (2017a) a étudié les différentes configurations d'équipes doctorales pour l'encadrement des étudiants, comme alternative à la relation dyadique, parfois limitative. Elle suggère que davantage d'attention soit accordée à la supervision afin de développer une approche holistique qui nourrit l'identité du doctorant. Pour sa part, Watts (2010) considère qu'il y a des avantages et des risques associés à l'encadrement en équipe. Cependant, l'auteure suggère que les points de tension liés à des perspectives conflictuelles peuvent être avantageux pour l'ensemble des membres de l'équipe lorsqu'ils sont abordés dans une ambiance d'entente. 
20 Dans le premier mode de travail en équipe, la dyade, l'étudiant travaille principalement avec le directeur de recherche alors que les codirecteurs ont un lien plus ténu (Robertson, 2017a). Le pouvoir est concentré dans les mains du directeur, et sa manière d'exercer ce rôle peut parfois limiter le pouvoir de l'étudiant dans sa capacité d'agir.

Figure 1. Mode dyade de facto (Robertson, 2017a, p. 365)'

Image

1034391C000080E100007E430289D011C3A83551.emf

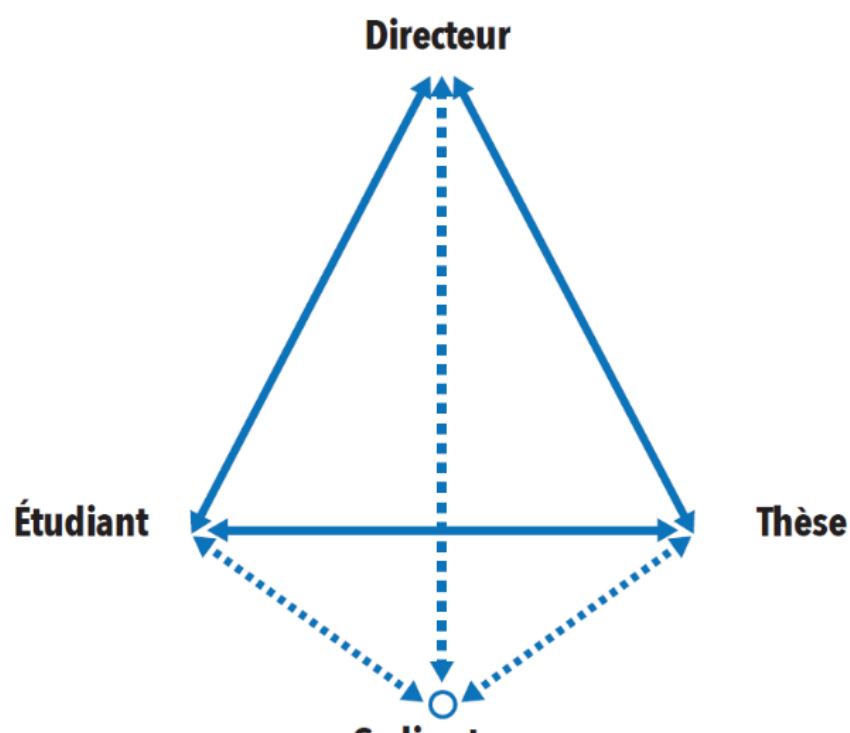

Codirecteur

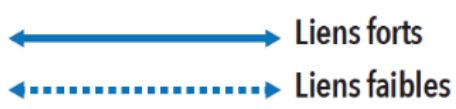

21 Dans le second mode de travail, la supervision segmentaire, le lien entre le directeur, l'étudiant et le projet demeure prédominant alors que la collaboration avec les autres superviseurs est épisodique et morcelée, avec des liens faibles. "The student is positioned as the central link, negotiating advice from a number of sources which may at times be contradictory" (Robertson, 2017a, p. 364). Dans cette perspective segmentaire, l'étudiant a plus d'occasions et de capacités d'exercer son pouvoir à travers la négociation et l'intégration des différentes sources d'informations. 
Figure 2. Mode segmenté (Robertson, 2017a, p. 366) ${ }^{2}$

Image

102F8478000074C000007E672AF5A04BEFEB45CC.emf

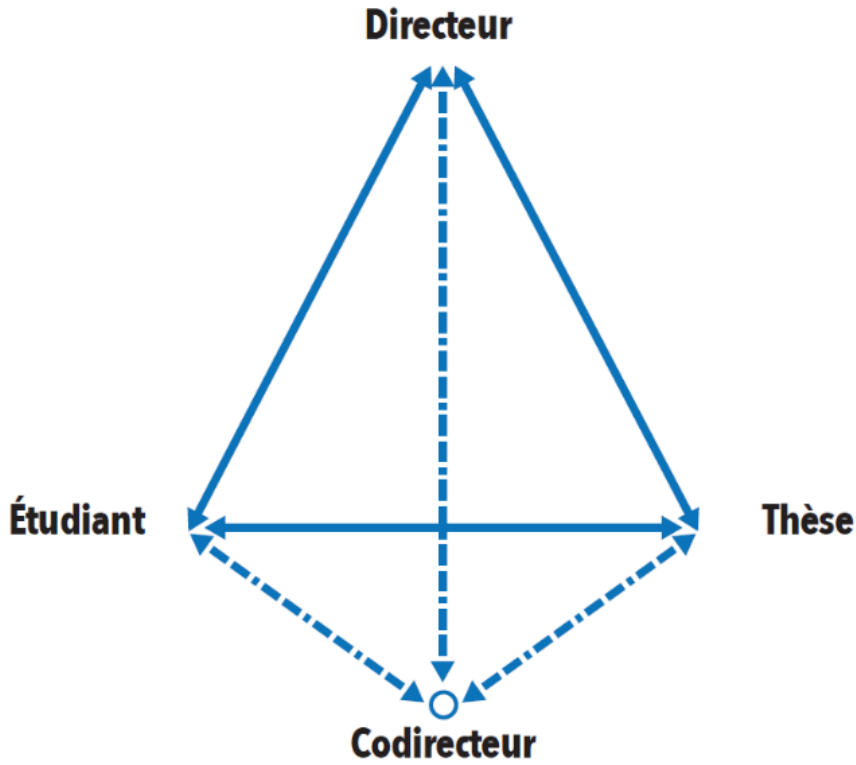

Liens forts

ヘーー・ーー・ーー・ーー Liens faibles / Liens épisodiques

22 Le troisième mode de travail est celui de l'équipe collaborative. Guerin et al. (2011) y proposent deux formes de supervision : l'une hiérarchique dans le cadre de laquelle le directeur joue un rôle central, l'autre horizontale qui renvoie plutôt à un pouvoir partagé au sein des membres de l'équipe. Le doctorant est explicitement partie intégrante de l'équipe "rather than being subject to supervision, and the work mediated though collaborative discussion » (Robertson, 2017a, p. 366). 
Figure 3. Mode collaboratif hiérarchique (Robertson, 2017a, p. 368) ${ }^{3}$

Image

10161AF800005A4900004BF4CE3D81B68ADC4D44.emf

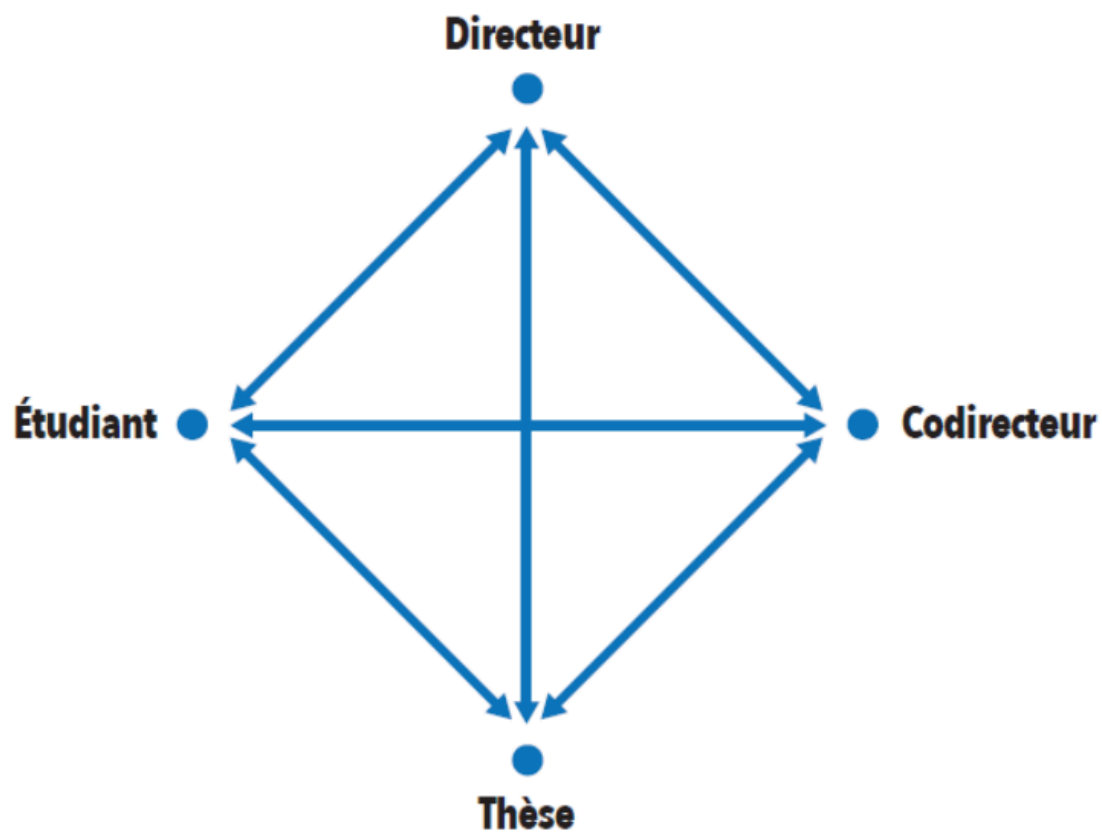

\section{Liens forts} différents membres de l'équipe gravitent autour en ayant des liens entre eux. Les différentes configurations relationnelles de l'équipe peuvent varier au cours du processus selon les circonstances. 


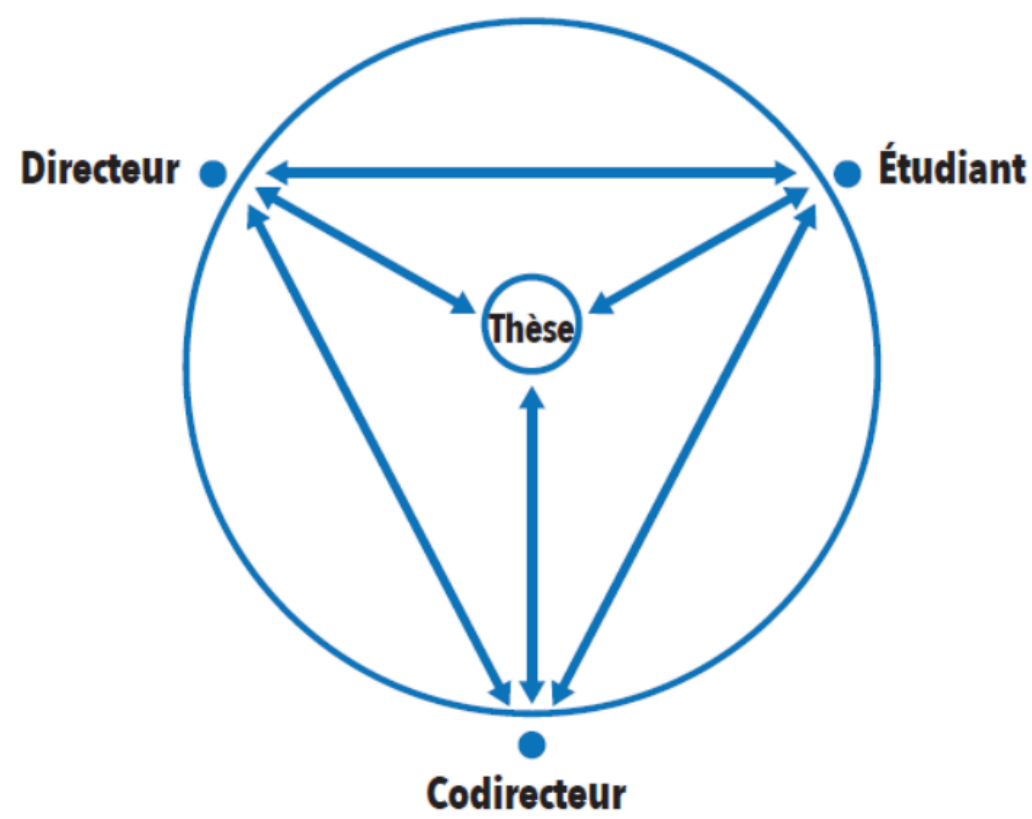

\section{Liensforts}

Dans l'un ou l'autre de ces cas de figure, le « pouvoir sur » et le " pouvoir à » travaillent ensemble et sont disponibles pour tous les modes de travail en équipe. Le " pouvoir avec » est plus propice dans le mode collaboratif horizontal dont le but est la création de connaissances. À la suite de cet exposé sur les différents concepts au cœur de cet article, la méthodologie s'y rapportant est présentée.

\section{Méthodologie}

La doctorante engage un retour réflexif sur le processus d'encadrement vécu lors de la réalisation de sa recherche doctorale, plus spécifiquement lors de la démarche d'analyse des données. Ce retour réflexif avait pour but de mieux saisir le sens caché des dynamiques relationnelles entre la doctorante et son équipe. Pour documenter ces dernières, une démarche de cueillette d'informations a été réalisée de janvier à mai 2017. Plusieurs étapes ont été franchies dans un ordre chronologique. Celles-ci rendent compte du processus d'encadrement collaboratif.

Premièrement, trois séances d'auto-explicitation (Vermersch, 2007) ont été réalisées par l'étudiante entre le 13 mars et le 10 avril. Ces séances portaient sur deux à trois moments spécifiés vécu avec chaque collaborateur : son directeur, son codirecteur et sa codirectrice. La visée d'explicitation était tout indiquée, car elle donne potentiellement accès à la face cachée de l'expérience. "Notre expérience subjective nous est aussi familière qu'un paysage, mais ce qu'un géologue, un géographe, un botaniste, etc. pourraient nous aider à y reconnaître est à la fois déjà là devant nos sens et en même temps invisible» (Vermersch, 1999, p. 15). Cet entretien installe les conditions afin que la personne ait accès à son vécu, en y retournant par la mise en évocation : « un acte 
mental s'accompagne d'un contenu représenté de façon quasi sensorielle" (Vermersch, 2010, p. 196). Il s'appuie sur une posture phénoménologique, laquelle est en quête d'un point de vue à la première personne (où le sujet se questionne lui-même, en auto-explicitation) ou d'un point de vue à la deuxième personne (où l'intervieweur questionne le sujet sur son expérience) (Lachance, 2016). Ce type d'entretien cherche le passage d'information entre la conscience pré-réfléchie vers la conscience réfléchie.

Il s'agit d'un questionnement à vide, sans contenu pour simplement permettre à la personne de rester sur ce moment et l'aider à le pénétrer, le décrire et le qualifier avec plus de finesse dans le déroulement du temps. Une séance d'auto-explicitation peut se dérouler, assise confortablement en s'adressant à soi-même : "Je te propose, si tu es d'accord, de prendre le temps de laisser revenir un moment de la cocréation du deuxième chemin d'analyse. Quand il sera présent, je t'invite à me le décrire. " Et lorsque le moment remonte, je fais la description de l'action entourant ce moment par l'écriture et je continue de me questionner pour fragmenter l'expérience dans le temps. Dans un deuxième temps, je peux questionner les couches de vécu (corporel, intérieur, affectif ou cognitif) : "Qu'est-ce qui se passe dans mon corps lorsque mon codirecteur me questionne sur le dénominateur commun? Est-ce que je me dis quelque chose?».

Deuxième, un entretien de groupe réunissant l'étudiante et son équipe sur l'analyse de leur pratique de collaboration et une période d'explicitation de groupe (Crozier, 2013) a été effectué le 12 avril (un directeur, deux codirecteurs et l'étudiante). Afin de rendre l'implicite plus explicite, l'entretien de groupe a été animé par une tierce personne qui possède une double expertise: dans l'analyse de la pratique et dans l'explicitation. Cette personne a eu préalablement accès aux contenus des premières séances d'autoexplicitation. L'animateur a demandé sur base volontaire aux trois collaborateurs d'écrire en style télégraphique: les idées, les pensées, les images qui leur viennent lorsqu'ils se laissent habiter par le thème "La collaboration de recherche dans l'analyse». En plus, il leur a été demandé d'identifier quelques moments qu'ils reconnaissent comme de la collaboration. Par ailleurs, l'entretien d'explicitation peut s'adresser autant à l'ensemble des personnes qu'à une seule personne du groupe (Crozier, 2013).

29 Troisièmement, une écoute de l'enregistrement de l'entretien de groupe a été faite par l'étudiante le 17 avril. Cette écoute a permis de revisiter les étapes importantes de l'encadrement collaboratif et l'information contenue.

30 Quatrièmement, deux séances d'auto-explicitation supplémentaires, le 24 et 25 avril ont été produites par l'étudiante à la suite de l'entretien de groupe sur des moments clés ciblés par le groupe en lien avec le point de bascule vers l'autonomie.

31 Cinquièmement, quelques entretiens informels avec le collaborateur de recherche, soit l'un des codirecteurs pour la présentation des résultats ont permis d'enrichir la réflexion.

Sixièmement, des échanges de courriels avec un professeur d'un groupe de recherche en psychosociologie de l'Université du Québec à Rimouski (UQAR).

Nous avons procédé à l'examen des données empiriques sous un angle phénoménologique, pour cette contribution, afin de décrire l'expérience vécue dans le processus de formation aux études doctorales. Un récit phénoménologique a été écrit et, comme le signalent Paillé et Mucchielli (2005), « nombre de situations de recherche 
ne requièrent pas autre chose que cette exposition fidèle et détaillée du monde vécu » (p. 76). La prochaine section explique le contexte de la recherche doctorale.

\subsection{Antécédents et contexte de la recherche doctorale}

L'étudiante était à l'origine diplômée du secteur de l'administration des affaires (1994). À la suite d'une formation en fasciathérapie $(1997,2001)$, elle décide ensuite de faire un diplôme d'études supérieures spécialisées en pédagogie perceptive du mouvement à Lisbonne (2002). Elle s'inscrit ensuite au doctorat en éducation à l'Université de Sherbrooke à temps partiel. Le processus doctoral en éducation s'est échelonné sur une période d'une dizaine d'années. Il a été ponctué par différentes étapes (voir figure 5). Le projet réalisé visait à identifier et à décrire les effets perçus dans la sphère personnelle (rapport au corps, à la présence et à leur santé) et professionnelle (conception de la santé et rapport aux autres professionnels de la santé et aux patients) de médecins ayant été formés à une approche Corps et Esprit, appelée pratiques du Sensible (Bois, 2007). Cette pratique comporte quatre outils principaux : 1) l'approche manuelle; 2) la gymnastique sensorielle; 3) l'introspection sensorielle et; 4) l'entretien verbal à propos de l'expérience corporelle. Une enquête a été réalisée au moyen de deux types d'entretiens : l'entretien compréhensif (Kaufmann, 2011) et l'entretien d'explicitation (Vermersch, 2010). À la suite d'une discussion avec son équipe d'encadrement, l'étudiante a construit deux démarches d'analyse : 1) la théorisation ancrée (Paillé, 1994) et 2) la création d'une liste de 26 phénomènes émergeants dans la majorité des entretiens faits auprès des participants. La prochaine section présente les résultats $d u$ processus réflexif lié aux grandes étapes du doctorat et à la relation d'encadrement.

Le directeur de thèse connaît bien la pratique étudiée, ce qui a constitué un avantage de taille pour l'intercompréhension. Chercheur chevronné, il a maintenu la cohérence à la fois dans le processus formatif et dans le projet de thèse. Le premier codirecteur était à la fois curieux et intéressé par la pratique étudiée, tout en étant critique à son égard, une double posture très intéressante. Son apport a permis d'enrichir la multiplicité des regards. À la suite de l'examen doctoral, dont elle a fait partie en juillet 2007, une deuxième codirectrice a été associée au projet. Il s'est avéré qu'adjoindre une personne du milieu médical était le meilleur moyen de faciliter l'acceptabilité du projet dans ce domaine réputé hermétique. 
Figure 5. Représentation temporelle du processus doctoral

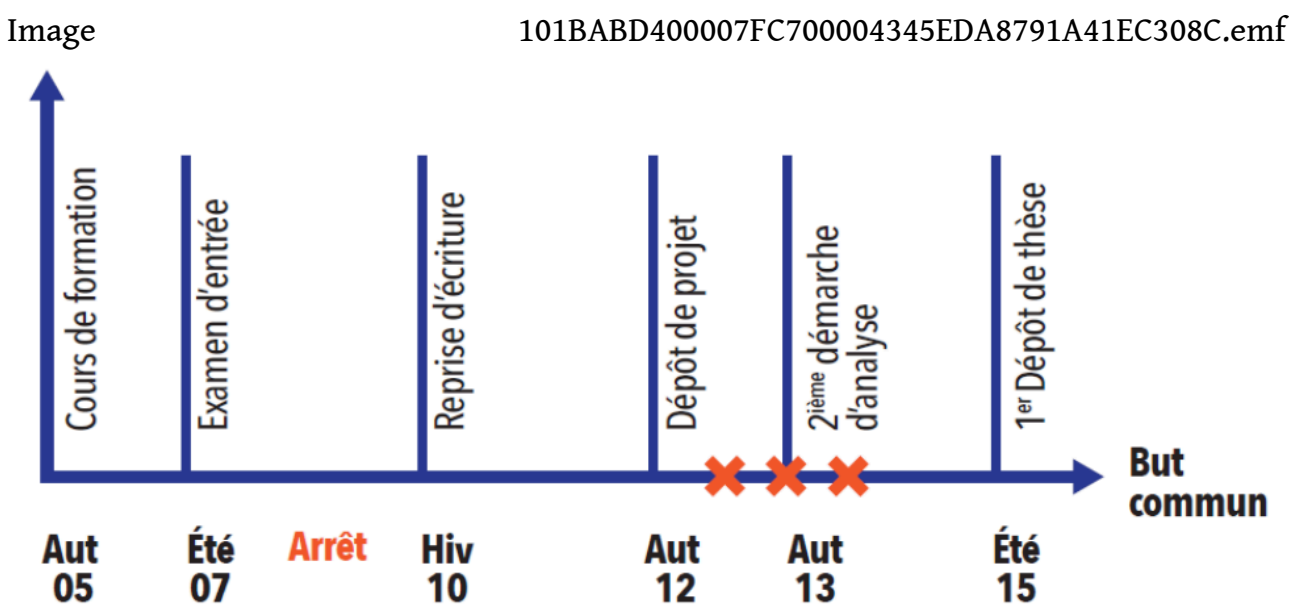

\section{X - Point de bascule}

L'examen de doctorat a été un jalon dans le parcours de l'étudiante, lui permettant de prendre conscience de certains écarts à combler dans la poursuite de ses études. Après l'examen doctoral de l'été 2007, et jusqu'au printemps 2010, l'étudiante a poursuivi ses lectures tout en ayant soin de consolider son rapport à son monde intérieur par des rencontres répétées, à titre d'étudiante libre, avec l'équipe de psychosociologie de l'UQAR. Toutefois, le projet doctoral a été concrétisé entre le printemps 2010 et le printemps 2016, date du dépôt final (Lachance, 2016; Lachance, Paillé, Desbiens et Xhignesse, 2016).

\section{Résultats}

Les résultats sont présentés en trois sections : 1 ) les phases de collaboration importante $\mathrm{du}$ doctorat; 2) les caractéristiques de l'encadrement collaboratif, et finalement; 3) les conditions qui ont permis de mener au point de bascule.

\subsection{Phases de collaboration importantes du doctorat}

Pour rendre compte des expériences de collaboration au sein de l'équipe d'encadrement, nous nous appuyons sur le cadre présenté plus haut. La suite de l'article est écrite à la première personne pour relater l'expérience de l'étudiante. Parfois elle est au pluriel pour l'expression des deux auteurs.

\subsubsection{Phase d'apprenti}

Au début de ma formation doctorale, entre l'année 2005 et 2007 et après mon examen de synthèse, j'ai l'impression de me sentir écartelée au sein de mon équipe d'encadrement dont les trois membres provenaient d'horizons multiples. À ce stade, les relations étaient dyadiques entre moi et chacun des différents membres de l'équipe. Il y avait peu de moments de rencontre avec toute l'équipe et très peu d'interactions entre les différents membres, tel que le suggère la figure 6. J'étais la principale cible des interactions. Je prenais l'ensemble des informations proposées par mon équipe, avec 
peu d'orientations liées spécifiquement à mon projet de recherche, tout en partageant mon opinion sur les thèmes discutés.

Figure 6. Écartèlement de la doctorante entre les trois membres de l'équipe

Image

1018BEE800007E1F00003D110515DFB94247D6B3.emf

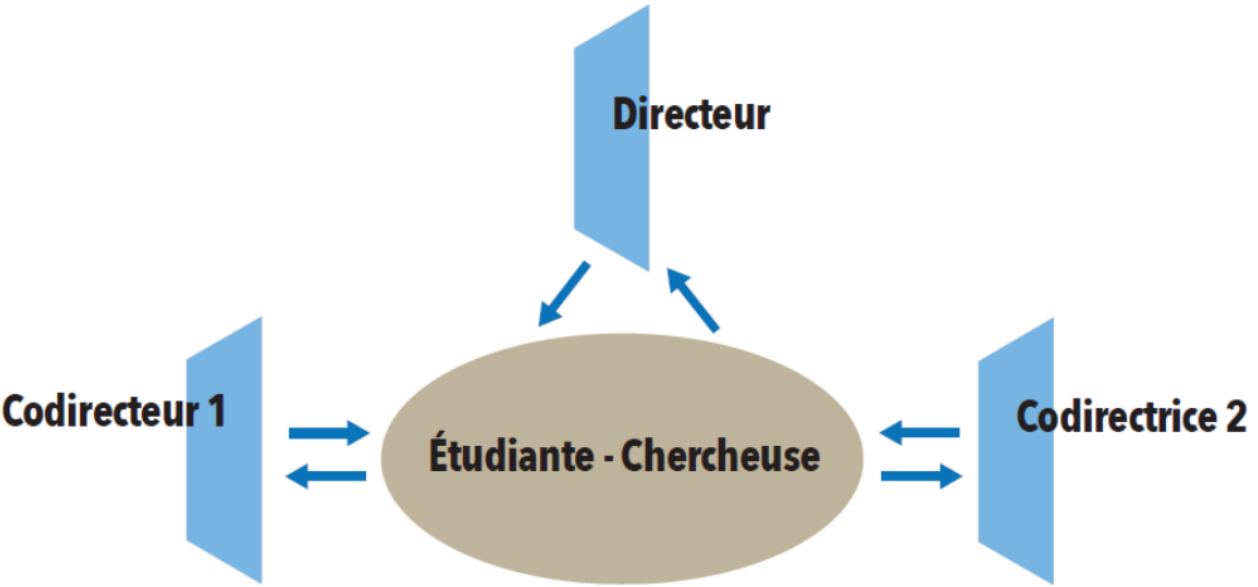

Dans cette période de la phase d'apprenti, le projet était peu défini. La problématique prenait difficilement la forme d'un entonnoir permettant de cibler l'objet spécifique de la thèse. Dans la réception d'autant d'informations, j'avais du mal à discerner ce qui permettrait d'élaborer mon projet de recherche, car je me sentais submergée et sans repère précis pour décider des directions à prendre. Dans la phase d'apprenti, chaque nouvelle orientation de lecture était une occasion de plus pour me perdre dans la documentation. Si cette étape a été riche pour l'élargissement de ma culture générale et spécifique à mon champ de recherche, je n'étais pas encore "née " comme chercheuse et je manquais de recul quant à l'objet de ma thèse.

\subsubsection{Phase intermédiaire de transformation}

Entre la phase d'apprenti et de compagnon, il y a eu une phase intermédiaire de transformation importante. Cette période s'est étendue entre le printemps 2010 et l'automne 2012, de l'écriture des premiers chapitres jusqu'au dépôt du projet. Cette phase a été marquée par certains moments difficiles, issus de la confrontation par le regard critique de personnes extérieures qui projetaient et éveillaient en moi une sensation d'impuissance et d'incapacité. La transformation du sentiment d'impuissance en un horizon de possibilités s'est produite de façon graduelle. Cette mutation a été le déclencheur d'une verticalité naissante définissable comme la capacité de faire face à la situation sans complaisance tout en ayant les deux pieds dans la réalité du moment, avec la sensation d'avoir du pouvoir sur les événements. Cette étape a été caractérisée par une auto-reconnaissance de ma capacité à conduire ma recherche et par une confiance en soi de plus en plus ancrée et moins ébranlable.

Cette sensation de confiance s'est tout d'abord construite au fil des rencontres successives, avec des membres du département de psychosociologie d'UQAR, au sein du programme de maîtrise où la posture de recherche se conjugue radicalement au «JE $»^{5}$. Le regard bienveillant des formateurs sur le germe en devenir des étudiants a été un ingrédient essentiel à ma construction intérieure, ce qui m'a permis de m'enraciner 
pour ensuite me verticaliser. Je me suis édifiée à partir d'un juste dosage: 1) d'expérience d'introspection sensorielle au travers de la pratique de la Méthode Danis Bois, plus récemment désignée par l'appellation méditation pleine présence (Bois et Eschalier, 2019); 2) de réflexions; 3) de périodes d'écriture et 4) du dialogue au sein de la communauté apprenante du programme de maîtrise. Celle-ci a aussi joué un rôle central de "contenant", je me trouvais alors en phase de transition identitaire et je ressentais régulièrement des états de fragilité intérieure. La posture préconisée par les enseignants du département de psychosociologie a été en fait le choix radical de poser son regard sur ce qui pousse, ce qui veut vivre, ce qui tend vers l'ouverture des possibles, plutôt que de centrer son regard sur ce qui tremble, crampe et résiste chez l'apprenante ${ }^{6}$.

43 Mon sentiment de confiance s'est ensuite consolidé grâce à des rencontres avec mon équipe d'encadrement. D'abord apparue comme une sensation fragile et fugace, la confiance s'est installée au fil du temps de façon durable. Cela m'a permis de m'édifier et de mieux définir les contours de mon projet de recherche. En tenant compte des directives de mon équipe d'encadrement, j'ai emprunté un processus qui était guidé et alimenté par des indications intérieures. Je vivais parfois des épisodes de négociation multilatérale entre les propos tenus par différents membres de mon équipe et ma propre guidance intérieure.

Durant cette même période, j'ai vécu des moments de fractures. À titre d'exemple, je m'engageais régulièrement dans des épisodes de confrontation bienveillante avec les membres de mon équipe, ce qui m'a conduite à bien distinguer au sein de mes écrits les différents acteurs qui prenaient la parole en moi : étudiante-militante, étudiantepraticienne, étudiante-expérientielle des pratiques du Sensible et l'étudiantechercheuse. Le défi était de taille! En effet, j'ai dû prendre conscience de ces différentes postures et apprendre à bien les distinguer, sans toutefois me sentir obligée de renier une part de moi.

Un deuxième moment clé de fracture s'est produit à l'automne 2010, à la suite de la rétroaction du directeur principal sur la première version du projet de thèse. Lors de cette rencontre décisive, le retour formulé par le directeur a mis en lumière l'ampleur des obstacles qui se dressaient dans la poursuite de mon projet, autant du point de vue de l'écriture que de la capacité à emprunter le processus d'entonnoir caractéristique à la problématique. La perception d'impuissance du directeur, face à l'ampleur du fossé à combler sur le plan de mes compétences, a trouvé écho en moi. En outre, le directeur a précisé, par rapport à cette situation de crise, que les importantes difficultés d'écriture que je connaissais l'ont amené à s'interroger sur la nécessité même que je fasse une thèse de doctorat. La réponse était pour lui négative, la vie comprenant tant d'autres aventures intéressantes à vivre. Ce positionnement franc de la part de mon directeur m'a conduite à me repositionner et à réaffirmer mes choix.

J'ai alors amorcé un revirement intérieur, devenant davantage actrice de mon processus, sans me perdre dans l'impuissance que j'éprouvais. En effet, j'ai demandé à mon directeur de me montrer précisément les problèmes dans le texte pour mieux saisir l'obstacle à franchir. De plus, cet épisode m'a obligée à faire des deuils en rapport avec le contenu de la thèse. Avec du recul, cet événement a été critique dans ma décision de continuer la démarche du doctorat, un tournant important alors que je fais le choix délibéré de continuer de m'investir, de faire des deuils, de reprendre plusieurs chapitres à la suite des commentaires de mon directeur. À partir de ce moment, les axes 
à suivre étaient très clairs et connectés à mes états intérieurs. Accompagnée par quelqu'un du domaine de la littérature, j'ai développé de nouvelles aptitudes en matière de rédaction. J'ai appris à ralentir mon état intérieur. Ainsi, ma pensée plus lente a permis que l'écriture puisse suivre. Dans cet état de présence sensorielle, il était plus facile de sentir les ruptures de texte, les phrases trop longues, les sujets coupés de leur verbe et la juste place des virgules.

\subsubsection{Phase de compagnonnage}

À cette étape, autour de la période du dépôt de projet en 2012 et de la période d'analyse des résultats en 2013, j'ai senti que je me redressais progressivement. Un axe vertical me traversait et me permettait de prendre racine. Cette verticalité naissante était, au début, peu perceptible. Il m'a fallu attendre un certain degré de redressement pour que j'arrive à le percevoir et à en sentir les effets dans mon interaction avec mon projet et mon équipe d'encadrement. Une sensation de confiance était de plus en plus perceptible, une confiance qui devenait de plus en plus solide. Ainsi, l'écartèlement est devenu un axe vertical faisant apparaître au fur et à mesure une forme conique représentée à la figure 7 .

Figure 7. Processus de verticalisation

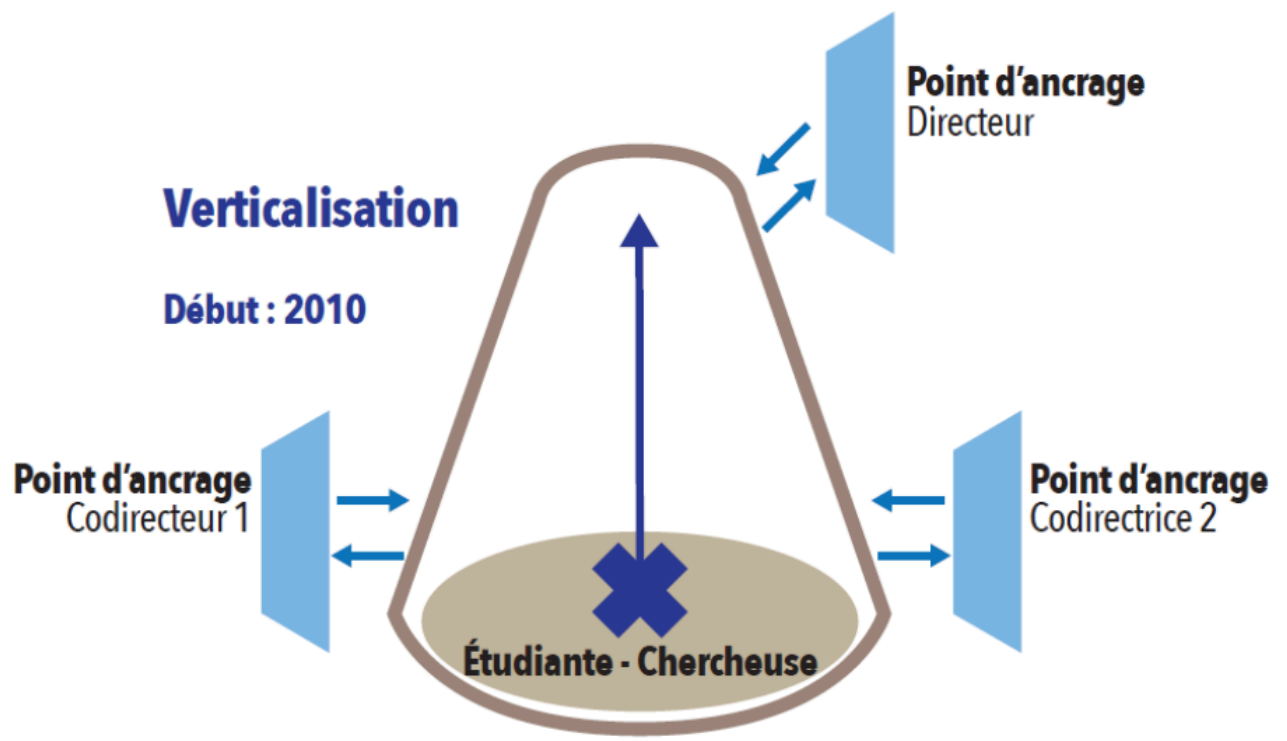

Dans cette nouvelle posture de la phase de compagnon, le processus de verticalisation s'est bien enclenché. Ce processus d'édification à la verticale était mû par la sensation de confiance en mes capacités de réaliser ma recherche. Dans cette phase, je me construis en tant que personne humaine et en tant que chercheuse. J'ai des opinions, j'assume et j'agis en tenant compte de mes convictions intérieures et des commentaires de mon équipe d'encadrement. Je sens plus clairement la direction à prendre pour mon projet de recherche et je suis à même d'argumenter mes points de vue lorsque c'est nécessaire. Il existe alors un lien entre l'information reçue et ma capacité d'en faire quelque chose de construit, par exemple lors de l'écriture de la problématique. Je discerne de mieux en mieux l'information pertinente dans les discussions avec mon 
équipe. Je donne une direction à mon projet et je cible mieux la personne de mon équipe d'encadrement la plus à même de répondre à mes besoins.

\subsubsection{Vers la phase du maître}

49 Le point de bascule pour m'engager dans mon processus d'autonomisation durant l'analyse s'est opéré à trois moments que je vais décrire en ordre chronologique, entre le printemps 2013 et le printemps 2014.

50 - Pré-mouvement vers l'autonomie

51 Après avoir analysé le contenu des deux premiers entretiens par thèmes et ensuite effectué des regroupements par catégories répertorié par rubriques et sous-rubriques, j'ai pris l'initiative d'écrire deux mémos analytiques. L'écriture de ces mémos a constitué une initiative personnelle dans ma démarche d'analyse et m'a permis d'avoir accès à une histoire de fond qui a influencé la suite de mon analyse, par exemple pour développer une structure commune par rubriques et sous-rubriques. Je présente plus bas les résultats d'une auto-explicitation autour de ce premier moment spécifié pointé par mon équipe, suite à l'entretien de groupe, point 4 de la démarche de cueillette d'informations.

52 Au fur et à mesure que j'écris, je réalise la facilité que j'ai à écrire les mémos, comme si tout se suivait et décrivait une histoire sans que j'aie quoi que ce soit à faire.

53 Je me sens à la limite observatrice d'un processus qui se déroule. J'en suis éblouie et tout étonnée à la fois. Une histoire déjà tout agencée de l'expérience de formation aux pratiques du Sensible d'un médecin, débordant sur son expérience de vie de manière plus globale.

54 Après la rédaction des deux mémos, je constate chez les deux participantes des mouvements similaires avec des contenus différents. Il y a des choses difficiles, un processus de prise de conscience et des changements dans leur vie personnelle et professionnelle.

55 - Point de bascule vers l'autonomie

56 Le point de bascule d'une condition de dépendance vers une autre d'autonomie est rencontré lors d'une discussion sur l'analyse des résultats avec mon codirecteur vers le début de l'automne 2013. Lors de cet échange émerge le potentiel d'un deuxième chemin d'analyse, que j'ai élaboré et exploité dans les étapes subséquentes. Voici les fruits d'une auto-explicitation autour de ce deuxième moment spécifié, point 4 de la démarche de cueillette d'informations.

57 Mon codirecteur et moi sommes à l'écoute et en réflexion. Il y a des interactions et une connexion vivante et dynamique. Et mon codirecteur me pose une question du genre : est-ce qu'il y a un dénominateur commun?

Point de bascule : Je m'assois, je me recentre intérieurement avec une sensation accrue des contours de mon corps et une augmentation de mon tonus général. Je suis centrée sur la tâche. L'information jaillit avec intensité. Je sens une vive énergie, une sensation d'ébullition, tel un volcan qui entre en éruption.

59 D'abord, je nomme facilement les dénominateurs communs dont je suis certaine. Ensuite, je me laisse habiter par un phénomène observé chez les participants. Je fais alors le tour de chaque participant, tel un radar, pour m'assurer de la présence du 
phénomène en question. Je considère un participant à la fois et je vais voir dans le contenu de l'entretien.

Nous sommes en train de cocréer quelque chose d'important. Je sens une forte connexion entre mon codirecteur et moi. Une connexion qui propulse et fait émerger ce qui est prêt, mûr.

61 Lors de l'expérience de cocréation, j'étais dans un état de fébrilité tout en étant habitée par la confiance en notre relation et par une sensation que l'expérience était contenue. La question formulée par mon codirecteur - à propos d'un dénominateur commun - a eu pour effet de déclencher l'intention, et j'avais l'impression d'être dans un haut niveau d'attention et d'engagement tout en étant en rétroaction avec mon codirecteur. Le temps n'existait plus et l'expérience était intense. J'étais dans une qualité de connexion avec moi et avec mon codirecteur, une forte connexion à partir de laquelle le processus a pu émerger. C'était une sensation de vivre l'expérience, mais de l'intérieur, comme si l'ensemble des verbatim des entrevues réalisées se trouvait inscrit sur les tissus de mon corps. Cette expérience m'a confirmée en tant que chercheuse. En effet, j'ai eu la sensation que je pouvais contribuer à la démarche, que mes intuitions avaient de la valeur et que j'avais la capacité de cocréer une démarche avec mon originalité.

- Engagement sur le chemin de l'autonomie

63 À la suite de cette rencontre avec mon codirecteur, je suis retournée chez moi poursuivre l'énumération des dénominateurs communs présents dans la majorité des entretiens. Ensuite, j'ai poursuivi ma démarche en élaborant un croisement entre les deux chemins d'analyse. Voici les fruits d'une auto-explicitation autour de ce troisième moment spécifique, point 4 de la démarche de cueillette d'informations.

64 Je suis présente à la comptable en moi, celle qui trouve toujours plusieurs chemins pour se rendre à la même réponse - afin de s'assurer de la qualité. Je tente la même expérience avec mon analyse. J'hésite un peu: puis-je utiliser une technique développée dans les examens comptables pour l'appliquer à l'analyse qualitative?

65 J'établis un croisement entre ma première et ma deuxième démarche d'analyse. Je reprends l'ensemble des citations prises pour chaque phénomène et je trouve les thèmes associés et la catégorie conceptualisante s'y rapportant. Je retourne aux différentes rubriques et sous-rubriques structurant les catégories pour cette personne en regardant ce qui manque comme catégorie liée à ce phénomène. Et là, je vais combler les lacunes.

66 Satisfaite, j'ai pu récupérer des citations liées à des phénomènes laissés pour compte. J'ai le sentiment que j'ai vraiment fait le tour et que rien n'a été oublié.

Je retourne consulter mon codirecteur, car je ne m'estime pas suffisamment légitime pour employer cette approche. Il la trouve intéressante et m'incite à faire tout le processus.

68 Ces trois moments spécifiques constituent les marches qui m'ont permis de franchir des pas par rapport à mon autonomie et à mon identité de chercheuse, d'accroître ma capacité à poser un regard critique tout en étant en mesure d'investir mon potentiel créatif au travers d'un processus d'appropriation. 


\subsection{Caractéristiques de l'encadrement collaboratif}

Les deux prochaines sections exposent les caractéristiques spécifiques de la collaboration au sein de l'équipe d'encadrement m'entourant: une collaboration de « seconde main » et une « dynamique vivante ».

\subsubsection{Une collaboration de « seconde main »}

70 À la suite de l'entretien de groupe, point 2 de la démarche de cueillette d'informations, nous avons constaté au fil de nos discussions que la collaboration au sein de l'équipe d'encadrement en était une de "seconde main». En effet, le directeur et les deux codirecteurs se sont rencontrés seulement quatre fois : à l'examen de synthèse, avant le dépôt du projet, lors de la présentation du projet et après la première vague d'entretiens. En fait, les différents collaborateurs avaient une relation essentiellement dyadique avec moi. Je faisais figure de carrefour giratoire, pour transmettre l'information de l'un vers les deux autres. La figure 8 illustre cette collaboration où j'occupais le centre. Cette position était potentiellement risquée, car l'information était concentrée entre mes mains pour être redirigée vers les personnes concernées.

Figure 8. L'étudiante comme carrefour giratoire de la collaboration

Image

10157C1C0000620100004419D4D4E236F8695DA9.emf

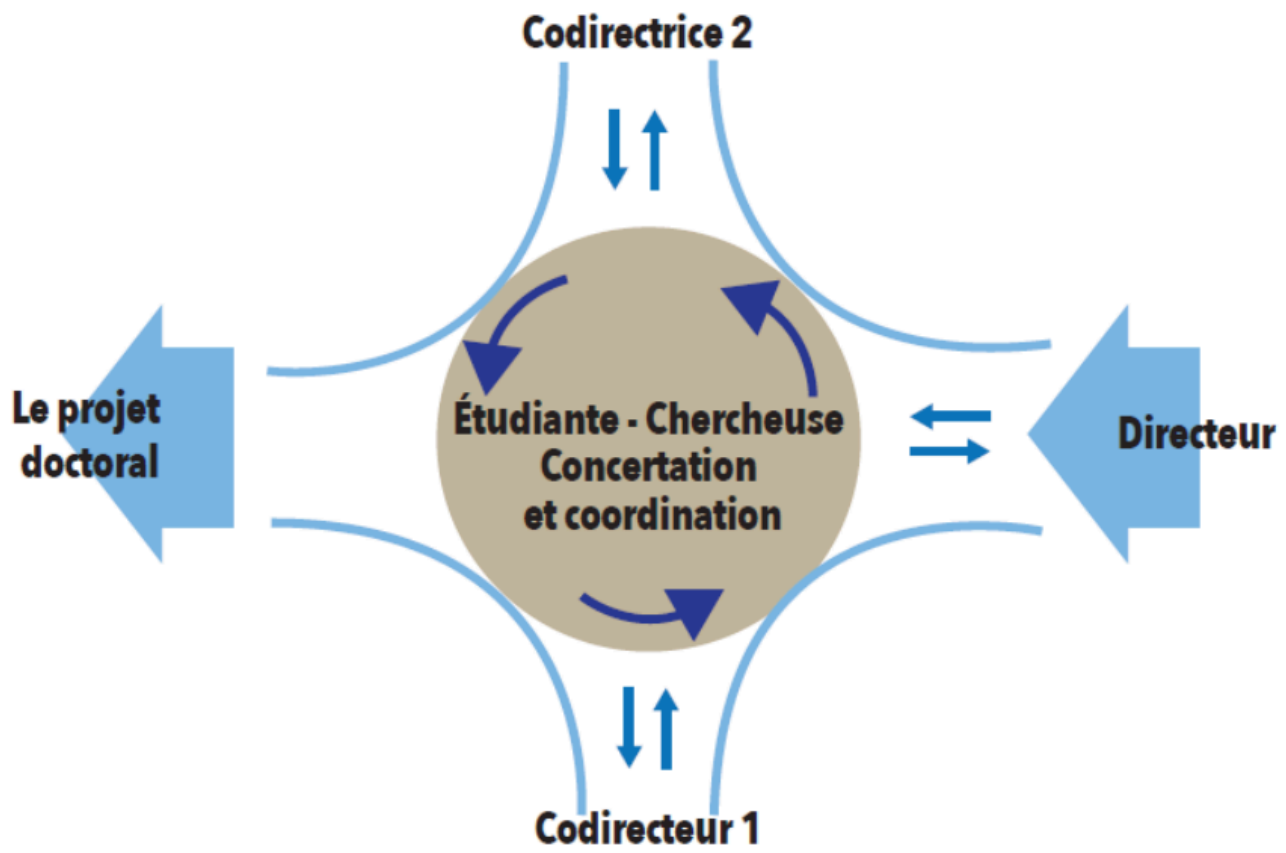

Dans la phase d'apprenti, les échanges tournaient parfois en rond, car je ne pouvais prendre position et voulais prendre l'information de chacun des collaborateurs. La posture de recherche des différents membres de l'équipe était très différente: un directeur de méthodologie en recherche qualitative, un codirecteur qui pratique la recherche à partir d'approches quantitatives et qualitatives et une codirectrice dans le milieu médical ayant plusieurs années d'expérience en recherche clinique. Cette situation a permis un grand nombre d'apprentissages, de nuances de point de vue, mais a aussi entraîné en premier lieu des écartèlements et des pertes de temps. Dans la 
phase de compagnonnage, la collaboration était plus fluide, riche de connaissances en raison de la diversité des expertises en place. Cela a été une période fructueuse : j'ai affirmé ma capacité à assumer davantage de leadership dans mon projet et, sous le coup de l'inspiration, j'ai produit en accéléré du contenu pertinent à la phase de l'analyse. J'ai bien profité de tous ces avantages tout en ayant plus de recul dans les prises de décision.

\subsubsection{Une collaboration « dynamique vivante »} d'encadrement était très dynamique. Ainsi, elle pouvait s'ajuster aux besoins et à la posture de l'étudiante de même qu'à l'évolution des différentes phases de la recherche. Si la collaboration était au départ asymétrique, elle est devenue par la suite plus symétrique et finalement asymétrique inversée. prenait plus de place pour me permettre d'acquérir une culture générale. Au commencement de la recherche, l'équipe alimentait beaucoup mes lectures et l'orientation à prendre. Dans cette première phase d'apprenti, je rencontrais plus souvent mon directeur de thèse lorsque les cours étaient terminés. Je rencontrais de manière moins régulière mon codirecteur. Un cours de tutorat avec lui à la fin de mon processus de formation a alimenté mes réflexions. Au cours de cette première partie, les rencontres avec ma codirectrice étaient peu fréquentes. celles de l'équipe étaient également productives et permettaient un enrichissement des points de vue. Après le dépôt des trois premiers chapitres, la phase sur le terrain permettant de procéder aux entretiens a été un moment durant lequel j'ai pris plus de place, même si j'étais toujours en apprentissage du processus d'analyse des résultats. Mon directeur était très présent dans les premières phases d'analyse afin de me permettre de bien saisir l'objet de chaque étape. Mon codirecteur prenait le temps de vérifier mes avancées à chacune des étapes, tandis que ma codirectrice a été présente à la fin pour la lecture des résultats, tout en suivant globalement toutes mes avancées.

Dans la dernière phase, après le point de bascule, j'ai pris les rênes de mon projet et je propulsais moi-même l'orientation à prendre dans l'analyse de mon projet. L'équipe d'encadrement était alors seulement consultée pour assurer l'adéquation de mes choix dans les processus d'analyse et de rédaction. Mon directeur et ma codirectrice faisaient la lecture des chapitres de résultats et de mise en perspective en enrichissant de leur point de vue mon travail. Avant le dépôt final, les trois membres de l'équipe ont relu ma thèse avec soin pour s'assurer de la cohérence et de la justesse des propos.

Selon les situations, la relation de collaboration était dans une forme de tension entre un pôle de supervision et un pôle d'accompagnement. À l'examen de synthèse, mon directeur, depuis un pôle d'accompagnement, a relevé les points forts, tout en notant les points à améliorer. Mes deux codirecteurs, pour leur part dans le pôle de supervision, étaient plutôt centrés sur les difficultés et les lacunes. Lors du retour sur mes premiers chapitres de thèse, mon directeur a adopté un rôle de supervision, tandis que mes deux codirecteurs étaient dans une relation de collaboration sous le signe de l'accompagnement. Je retiens qu'il est important de savoir reconnaître le bon moment

Revue internationale de pédagogie de l'enseignement supérieur, 37(3) | 2021 
pour changer de posture, même si parfois cela a pu être difficile pour les membres de l'équipe.

\subsection{Conditions nécessaires au point de bascule}

77 Mon passage au sein du programme de maîtrise dans les pratiques psychosociales a été un moment fondateur pour construire ma confiance en soi, par le regard bienveillant des formateurs. Les formateurs, tout comme le groupe, ont joué un rôle central de « contenant » dans ces périodes de transition identitaire.

Lors de l'entretien de groupe, point 2 de la démarche de cueillette d'informations, l'ensemble des collaborateurs s'accordait pour dire que l'ambiance dans l'équipe était de grande qualité et reflétait le respect. Il y avait une belle qualité d'écoute et de bienveillance, tout en respectant l'expertise de chacun. Cette ambiance a facilité, selon nous, l'engagement de chacun à s'exprimer et à s'adapter aux situations au fur et à mesure qu'elles se présentaient.

79 La précieuse collaboration entre tous les membres de l'équipe d'encadrement a fait en sorte qu'un accompagnement a été offert à l'étudiante, même au cours de certaines situations de crise et de tension. Des situations qui, par ailleurs, ont créé des conditions favorables à la collaboration. En effet, il arrivait parfois qu'un membre de l'équipe engendre une situation de crise et qu'un autre rende possible un accompagnement dans le processus. Le tout a été réalisé sous le signe de la médiation, en mettant plus de nuances dans les propos rapportés, et ce, afin de me permettre de mieux saisir les défis sans me dévaloriser. Comme les crises avaient pris forme au sein de la dimension relationnelle, c'est également là qu'elles se sont dénouées, dans la manière de dire les choses et de m'accompagner. La plupart du temps, les interactions s'effectuaient sur un plan horizontal. Toutefois, lors des moments avec un pôle dominant de supervision, il est arrivé que les relations suivent un rapport hiérarchique vertical : par exemple, lors du dépôt de mon examen de synthèse à l'été 2007 et lors de la première rétroaction sur mes trois premiers chapitres à l'automne 2010.

\section{Discussion conclusive}

80 La discussion conclusive aborde quatre thèmes : 1) les «positions de savoir » selon les phases de la collaboration; 2) la collaboration « dynamique vivante », 3) les conditions d'accompagnement et, enfin; 4) les limites et les retombées du présent projet.

\section{1. «Positions de savoir » selon les phases de la collaboration}

81 De manière générale, les "positions de savoir» ont varié dans mon processus d'encadrement. En effet, en début de parcours (phase apprenti, similaire à la posture "de conduite" avec le directeur "devant» Gérard (2009)), au moment où la collaboration était asymétrique, j'ai remis alors à l'équipe d'encadrement « un pouvoir plus grand dans le savoir de recherche à produire, en s'inscrivant dans un certain rapport de dépendance " (Morrissette et Desgagné, 2009, p. 140) suivant une certaine forme de hiérarchisation des positions. Je prenais rarement position dans les discussions et comme Guerin et Green (2015) l'on déjà mis en évidence, j'étais troublée par les retours différents des membres de mon équipe. À la fin de mon parcours 
doctoral, vers la phase du maître ou de « l'accompagnement-suivi » (Gérard, 2009) ou le directeur est "derrière », l'asymétrie s'est inversée, et je n'ai plus appréhendé de la même façon le jugement des autres, m'attribuant alors « un pouvoir de contribuer au savoir de recherche» (Morrissette et Desgagné, 2009, p. 140). J'ai orienté le projet en prenant les commentaires de l'équipe qui me convenaient (Guerin et Green, 2015). J'ai propulsé mon projet.

82 À certains moments, plus près du point de bascule, la «position de savoir " a varié selon les membres de l'équipe. Elle était égalitaire avec certains et plus hiérarchique avec d'autres. Cette période de bascule s'est déroulée durant l'analyse des données. D'abord, j'ai développé, avec mon directeur, chacune des étapes de la première démarche d'analyse, et ce, à l'intérieur des limites d'un espace pédagogique et validant. L'écriture des mémos a donné du corps à mon autonomie et a aussi contribué à enrichir l'analyse des résultats obtenus. Et puis enfin, la conception du deuxième chemin d'analyse a résulté d'un moment de cocréation en collaboration avec mon codirecteur. Cela a constitué un moment charnière qui m'a menée vers la phase du maître, vécu comme un état de flow, possible grâce à la non préoccupation de soi et au renforcement du sens de soi, conditions préalables décrites par Csikszentmihalyi (1990). Cette expérience a contribué à ma construction identitaire comme chercheuse. Nous retrouvons dans ce moment une construction de mon autonomie, voire de mon émancipation. Simultanément, il y a eu auto-reconnaissance de ma valeur et déploiement de ma capacité à mener mon projet avec créativité, propre à l'émancipation (Lee, 2008).

Il semble que chaque passage vers une nouvelle phase a été accompagné d'un moment critique, difficile à vivre ou non, et ce, sur une période de temps plus ou moins longue. En effet, les passages ne se sont pas effectués de façon instantanée, mais plutôt graduelle, et ce, sur plusieurs mois. Par exemple, durant la phase intermédiaire de transformation entre les phases d'apprenti et de compagnonnage, j'ai vécu un processus de différenciation de certaines co-identités (Vermersch, 2011) : étudiantemilitante, étudiante-praticienne, étudiante-expérientielle des pratiques du Sensible et étudiante-chercheuse. Cette période de confrontation bienveillante avec mon équipe m'a permis d'accéder à une liberté d'expression tout en gagnant en pertinence. Un deuxième exemple est le moment de crise à l'automne 2010. Cette crise, qui a eu l'effet d'un séisme, a été simultanément un tremplin. Les crises, comme la posture de carrefour giratoire, ont constitué des risques pour ma recherche. Le doctorat est un périple parsemé d'inconnu et d'incertitude (Turner, 2015). Ce moment a été critique et porteur d'une propension à la verticalité. De toute évidence, ces deux moments forts ont constitué des étapes importantes dans la construction d'une nouvelle posture : celle de la chercheuse.

\subsection{Collaboration «dynamique vivante »}

84 La collaboration "dynamique vivante » a permis de faciliter le dénouement des crises qui ont été vécues à travers la dimension relationnelle. Dans le parcours, les pôles de supervision et d'accompagnement étaient présents et souvent complémentaires, ce qui a permis de mieux naviguer lorsque des tensions étaient présentes. De plus, le respect de chaque membre de l'équipe en tant que personne, la reconnaissance de chacun pour l'expertise de l'autre a constitué, selon nous, des ingrédients de base du bon 
fonctionnement de l'équipe. Dans les moments de crise, l'autorégulation dont parlent Styles et Radloff (2001) a aussi permis de franchir des étapes déterminantes. Lors du retour de mon directeur, il y a eu une première régulation (hétéro) de mon directeur (pôle superviseur) vers moi, puis une deuxième régulation (auto) avec moi-même pour mieux établir mes choix et la marche à suivre pour la continuité, et ce, dans une posture métacognitive. Par la suite, lors de rencontres avec mes codirecteurs (pôle accompagnement), une troisième régulation s'est produite. Le pôle accompagnement a ouvert la porte à une médiation entre moi et le pôle supervision, ce qui a favorisé et enrichi la collaboration. Bourret (1997) valorise la nécessité d'intervenir, car « elle va pouvoir limiter et contrôler l'impression de "tourner en rond" que l'étudiant éprouve " (p. 392).

Tous les modes de travail en équipe proposés par Robertson (2017a) ont été expérimentés pour répondre à mes besoins et ceux du projet; la forme n'était donc pas figée. En début de parcours, le mode de travail dominant a été vécu comme une dyade et correspondait à la phase d'apprenti. Par la suite, il s'est apparenté à l'équipe segmentaire dans la phase de compagnonnage, car plus d'une personne m'a accompagnée. Enfin, dans la dernière ligne droite, tous les membres ont contribué selon un mode collaboratif horizontal, par leurs interactions avec moi dans la phase $\mathrm{du}$ maître. De par ces changements de fonctionnement dans l'équipe, la manière de distribuer le pouvoir a changé aussi. D'un « pouvoir sur » au début, il devient à la fin "un pouvoir avec", afin de maximiser les avancées de l'équipe (Robertson, 2017a). Cette danse relationnelle exprime bien la qualité d'adaptation qui a été requise, dans notre système d'encadrement et mon identité (Amundsen et McAlpine, 2009), afin de favoriser mon accompagnement.

Beauvais (2004) définit bien l'accompagnement comme un processus pour aider l'autre à se construire et lui permettre de devenir un sujet autonome et assumé. Vus sous cet angle, les objectifs de l'accompagnement cadrent bien avec les finalités du processus d'études supérieures. En effet, l'objectif ultime est de permettre à l'étudiant de devenir autonome et compétent dans sa capacité de conceptualiser et de produire une recherche, tout en ayant un regard critique sur différents courants théoriques et en étant à même de prendre position et de s'assumer (ADÉSAQ, 2018). La réflexion présentée dans le cadre de cette contribution expose bien mon cheminement vécu : le développement progressif d'une posture de verticalité, d'une capacité à prendre position et d'affirmer mon opinion dans mon parcours de thèse. La réflexion fait aussi état de la prise d'autonomie graduelle qui s'est effectuée au fil de temps et du point de bascule survenu dans l'analyse des résultats, moment clé au cours duquel j'ai pris le flambeau et j'ai avancé en donnant l'orientation à suivre.

\subsection{Conditions d'accompagnement}

87 Les conditions d'accompagnement sont en lien direct avec la capacité d'installer de la confiance, du respect et, lorsque c'est nécessaire, de m'offrir du « contenant » qui me permettent de mieux me construire à partir d'un espace en cours d'édification. Ces conditions m'ont permis une meilleure adaptation et le renversement de mon sentiment d'impuissance. Ainsi, j'ai franchi un cap qui m'a menée sur un chemin de construction identitaire (Gohier et al., 2001). Depuis lors, j'ai pu rencontrer des obstacles sans être immobilisée indéfiniment, ni submergée par l'impuissance, et j'ai su 
trouver comment rebondir plus rapidement. Il semble que l'état de confiance construit à différentes étapes du parcours grâce à des relations significatives (Robertson, 2017b) constitue un prérequis aux transformations identitaires (Gohier et al., 2001) et engendre le "sentiment du droit à exister, donc à créer» (p. 8). L'identité professionnelle peut prendre racine en amont sur les fondations de l'identité de la personne. L'état de confiance m'a permis de me laisser ébranler par les membres de mon équipe, et ce, pour mieux me construire en tant que chercheuse critique (Robertson, 2017b), et ainsi, mieux habiter les passages entre les différentes phases : apprenti, compagnon et maître.

La collaboration autour de mon projet de thèse a permis de rehausser la qualité du résultat et j'ai vraiment eu la sensation d'avoir contribué à une œuvre collective. Le processus a été profondément émancipateur. Comme le mentionne Reboul (1971), " éduquer, ce n'est pas fabriquer des hommes selon un commun modèle, c'est libérer en chaque homme ce qui l'empêche d'être soi, lui permettre de s'accomplir selon son génie singulier » (p. 43). Au-delà du fait de donner du sens à une pratique (Little, 1990), cette collaboration a contribué à orienter une vie en construction, à donner naissance à une personne capable de se faire entendre et de communiquer son point de vue, tout en étant ouverte à celui de l'autre, et ce, avec toute la rigueur scientifique.

\subsection{Limites et retombés du présent projet}

L'examen phénoménologique des données empiriques a des limites. Il est restreint par les frontières de la conscience de la personne et par la nécessité d'écouter les données avant d'être tenté de les «faire parler » (Paillé et Mucchielli, 2005, p. 70). Toutefois, il a été possible de repousser ces limites. Ainsi, l'entretien de groupe a permis d'enrichir l'information des multiples regards des membres de mon équipe d'encadrement, en plus d'être animé par une personne externe et neutre. Des entretiens répétés avec le collaborateur de recherche et l'évaluation des pairs ont confronté mon expérience et ont permis de l'expliciter davantage. La lecture de l'article par mon équipe a permis d'apporter des corrections de recadrage pour rendre l'expérience plus juste aux regards de tous. Aucune généralisation des résultats n'est possible, par contre la qualité de la description participe à leur transférabilité.

Les retombées touchent différents acteurs et l'aspect relationnel: 1) soutenir les étudiants dans la phase d'apprenti et leur permettre de mieux saisir leur inconfort face à l'errance et à l'hésitations du débutant, 2) éveiller la direction ou l'équipe d'encadrement sur la nécessité d'installer la confiance dans la relation, 3) mettre en évidence l'importance de la juste dose des pôles de supervision et d'accompagnement lors des crises qui peuvent devenir charnières dans le fait de continuer ou d'arrêter la formation au doctorat, 4) lors des moments de friction, comment le pôle d'accompagnement joue un rôle central de médiation entre le pôle supervision et l'étudiant et 5) dans les phases de tension, ouvrir le dialogue. Mon autonomisation est passée par donner du pouvoir aux membres de l'équipe par la confiance mutuelle. Celle-ci a été une source de résilience pour traverser les moments de friction générateurs d'idées nouvelles, sans éviter les opinions divergentes et les discussions animées (Robertson, 2017b) dont parfois les doctorants tentent de se soustraire (Guerin et Green, 2015). 


\section{BIBLIOGRAPHIE}

Amundsen, C. et McAlpine, L. (2009). Learning supervision: Trial by fire. Innovations in Education and Teaching International, 46(3), 331-342.

Association des doyens des études supérieures au Québec (ADÉSAQ). (2018). Les compétences visées dans les formations aux cycles supérieurs. 1-26.

Beauvais, M. (2004). Des principes éthiques pour une philosophie de l'accompagnement. Savoirs, 6(3), 99-113.

Bois, D. (2007). Le corps sensible et la transformation des représentations chez l'adulte [thèse de doctorat, Université de Séville].

Bois, D. et Eschalier, I. (2019). La méditation pleine présence. Les sept voies d'accès à la chaleur humaine. Éditions Eyrolles.

Bourret, A. (1997). L'encadrement des étudiants des cycles supérieurs : résultats d'une enquête effectuée à l'Université du Québec à Montréal. Dans L. Langevin et L. Villeneuve (dir.), L'encadrement des étudiants, un défi du XXI siècle (p. 376-414). Éditions Logiques.

Bravo, G., Saint-Mieux, J. et Dubois, M.-F. (2007). Health sciences graduate students' perceptions of the quality of their supervision: a measurement scale. Revue canadienne d'enseignement supérieur, 37(2), 69-88.

Brew, A. (2001). Conceptions of research: A phenomenographic study. Studies in Higher Education, 26(3), 271-285.

Crozier, J. (2013). Utiliser les techniques d'explicitation au sein d'un groupe. Explicitez, 99, 1-9.

Csikszentmihalyi, M. (1990). Flow : The psychology of optimal experience. Harper \& Row.

Desgagné, S. (1997). Le concept de recherche collaborative : l'idée d'un rapprochement entre chercheurs universitaires et praticiens enseignants. Revue des sciences de l'éducation, 23(2), 371-393.

Gérard, L. (2009). L'accompagnement en contexte de formation universitaire : Étude de la direction de mémoire comme facteur de réussite du Master [thèse de doctorat, Université Nancy II].

Gohier, C., Anadón, M., Bouchard, Y., Charbonneau, B. et Chevrier, J. (2001). La construction identitaire de l'enseignant sur le plan professionnel : un processus dynamique et interactif. Revue des sciences de l'éducation, 27, 3-32.

Guerin, C., Green, I. et Bastalich, W. (2011). Big love : Managing a team of research supervisors. Dans V. Kumar et A. Lee (dir.), Doctoral education in international context: Connecting local, regional and global perspectives (p. 138-153). University Putra Malaysia Press.

Guerin, C. et Green, I. (2015). They're the bosses': Feedback in team supervision. Journal of Further and Higher Education, 39(3), 320-335.

Jones, M. (2013). Issues in doctoral studies - forty years of journal discussion: Where have we been and where are we going? International Journal of Doctoral Studies, 8, 83-104.

Jutras, F., Ntebutse, J. G. et Louis, R. (2010). L'encadrement de mémoires et de thèses en sciences de l'éducation : enjeux et défis. Revue internationale de pédagogie et de l'enseignement supérieur, 26(1), $1-22$. 
Kaufmann, J.-C. (2011). L'enquête et ses méthodes : l'entretien compréhensif (3éd.). Armand Colin Éditeur.

Lachance, J. (2016). Étude exploratoire auprès de médecins des effets perçus d'une formation corps / esprit fondée sur les pratiques du sensible. [thèse de doctorat, Université de Sherbrooke]. https:// savoirs.usherbrooke.ca/handle/11143/8838

Lachance, J., Paillé, P., Desbiens, J.-F. et Xhigesse, M. (2016). Incidences des transformations du rapport au corps dans la sphère personnelle et professionnelle de médecins. Dans F. Vinit et J. Quintin (dir.), La place du corps dans la rencontre de soi et l'accompagnement (p. 163-199). Édition du Cercle interdisciplinaire de recherches phénoménologiques (CIRP).

Lee, A. (2008). How are doctoral students supervised? Concepts of doctoral research supervision. Studies in Higher Education, 33(3), 267-281.

Little, J. W. (1990). The persistence of privacy: Autonomy and initiative in teachers' professional relations. Teacher Collège Record, 91(4), 509-536.

Morrissette, J. et Desgagné, S. (2009). Le jeu des positions de savoir en recherche collaborative : une analyse des points de vue négociés d'un groupe d'enseignantes du primaire. Recherches qualitatives, 28(2), 118-144.

Narcy-Combes, J.-P. (2001). La recherche-action en didactique des langues : apprentissage, compagnonnage ou évolution libre? Recherche et pratiques pédagogiques en langues de spécialité, 21(2), 40-52.

Paillé, P. (1994). L'analyse par théorisation ancrée. Cahiers de recherche sociologique, 23, 147-181.

Paillé, P. et Mucchielli, A. (2005). L'analyse qualitative en sciences humaines et sociales. Armand Colin Éditeur.

Paul, M. (2002). L'accompagnement : une nébuleuse. Éducation permanente, 153(4), 43-56.

Reboul, O. (1971). Philosophie de l'éducation. Presses universitaires de France.

Robertson, M. J. (2017a). Team modes and power: supervision of doctoral students. Higher Education Research \& Development, 36(2), 358-371.

Robertson, M. J. (2017b). Trust : the power that binds in team supervision of doctoral students. Higher Education Research \& Development, 36(7), 1463-1475.

Styles, I. et Radloff, A. (2001). The synergistic thesis : Student and supervisor perspectives. Journal of Further and Higher Education, 25(1), 97-106.

Turner, G. (2015). Learning to supervise: four journeys. Innovations in Education and Teaching International, 52(1), 86-98.

Vermersch, P. (1999). Pour une psychologie phénoménologie. Psychologie française, 44(1), 7-18.

Vermersch, P. (2007). Bases de l'auto-explicitation. Explicitez, 69, 1-31.

Vermersch, P. (2010). L'entretien d'explicitation (6 éd.). ESF.

Vermersch, P. (2011). Note préparatoire sur le thème des co-identités. Explicitez, 90, 13-18.

Watts, J. H. (2010). Team supervision of the doctorate: Managing roles, relationships and contraductions. Teaching in Higher Education, 15(3), 335-339. 


\section{NOTES}

1. Copyright (C) HERDSA, reproduit avec l'autorisation de Informa UK Limited, agissant sous le nom de Taylor \& Francis Group, www.tandfonline.com au nom de HERDSA.

2. Copyright ( $)$ HERDSA, reproduit avec l'autorisation de Informa UK Limited, agissant sous le nom de Taylor \& Francis Group, www.tandfonline.com au nom de HERDSA.

3. Copyright $\odot$ HERDSA, reproduit avec l'autorisation de Informa UK Limited, agissant sous le nom de Taylor \& Francis Group, www.tandfonline.com au nom de HERDSA.

4. Copyright @ HERDSA, reproduit avec l'autorisation de Informa UK Limited, agissant sous le nom de Taylor \& Francis Group, www.tandfonline.com au nom de HERDSA.

5. Berger, E. (2009). Rapport au corps et création de sens en formation d'adulte [thèse de doctorat, Université Paris 8].

6. Rugira, J.-M. et Léger, D. (2016). De la vulnérabilité en accompagnement du changement humain : enjeux éthiques et défis pédagogiques. Ethica, 20(2), 89-106.

\section{RÉSUMÉS}

L'article relate le chemin parcouru par une doctorante durant sa formation encadrée par une équipe formée d'un directeur et de deux directeurs adjoints. Devant le défi présenté par cette démarche, la dimension relationnelle a joué un rôle prépondérant, mais souvent occulté. Le but de cet article est de retracer les étapes importantes et les caractéristiques de la collaboration vécue, avec un pôle de supervision ou avec un pôle d'accompagnement. Afin d'appréhender ce processus complexe, les concepts de collaboration et d'accompagnement sont développés. Une démarche de recherche documentaire a été réalisée pour compléter des séances d'autoexplicitation et d'entretiens avec l'équipe d'encadrement de la thèse. Le processus d'encadrement est présenté sous l'angle d'une collaboration en quatre phases, soit celles d'apprenti, de période intermédiaire de transformation, de compagnon et de maître (NarcyCombes, 2001). Il met en lumière les conditions qui ont favorisé le point de bascule vers l'autonomie tel qu'il a été vécu par l'étudiante au doctorat, ce qui a mené à sa construction identitaire comme chercheuse. La construction de la confiance et le respect ont été des conditions vers ma transformation identitaire et mon autonomisation. Les moments de crise ont été gérés à partir d'une médiation entre le pôle de supervision et d'accompagnement habité par les différents membres de l'équipe. Cet article offre une incursion, du point de vue de la doctorante et la chercheuse en devenir, en plein cœur de l'étroit processus de collaboration qui a pris place entre elle et les membres de son équipe d'encadrement.

The article describes the path taken by a doctoral student during her training supervised by a team composed of a director and of two assistant directors. Faced with the main challenge presented by this process, the relational dimension played a preponderant but often obscure role. The purpose of this article is to track down the significant steps and the characteristics of the collaboration experienced, with a supervision or an accompaniment pole. In order to grasp this complex process, the collaboration and accompaniment concepts are developed further. A documentary research process was conducted to complement self-explicitation and interview sessions with the thesis supervision team. The supervision process is presented from the angle of a collaboration in four phases: the apprentice, the intermediate transformation period, the 
journeyman and the master (Narcy-Combes, 2001). It highlights the conditions that promoted the tipping point towards autonomy as experienced by the doctoral student, which led to her identity building as a researcher. Confidence building and respect were the basic conditions towards identity transformation and autonomy. As crisis happened, they were managed from a mediation between the supervision and accompaniment poles embraced by different team members. This article provides an incursion, from the future researcher's point of view, in the heart of the narrow collaboration process which took place between her and the members of her supervision team.

\section{INDEX}

Mots-clés : collaboration, études doctorales, encadrement, direction de recherche, accompagnement

\section{AUTEURS}

\section{JOSÉE LACHANCE}

Université de Sherbrooke, Sherbrooke, Canada, lachancejoe@hotmail.com

\section{JEAN-FRANÇOIS DESBIENS}

Université de Sherbrooke, Sherbrooke, Canada, Jean-François.Desbiens@Usherbrooke.ca 